\title{
Do the predictors of right ventricular pacing-induced cardiomyopathy add up?
}

\author{
Harsha Perla ${ }^{1}$, Sirish Chandra Srinath Patloori ${ }^{2}$, David Chase ${ }^{3}$, and John Jacob ${ }^{4}$ \\ ${ }^{1} \mathrm{CMC}$ Vellore \\ ${ }^{2}$ Christian Medical College and Hospital Vellore \\ ${ }^{3}$ Flinders Medical Centre \\ ${ }^{4}$ Christian Medical College
}

September 10, 2020

\begin{abstract}
Introduction: Knowledge of factors causing pacing-induced cardiomyopathy (PICM) is incomplete. We sought to estimate the incidence and predisposing factors for PICM in South Asian population and evaluate if the risk they portend adds up. Methods: Consecutive patients with preserved LVEF undergoing pacemaker (PM) implantation between 2012 and 2018 were analysed. Results: A total of 749 patients (68.4\% male; mean age $59.2 \pm 14.08$ years) were included in the analysis. PICM developed in $74(9.9 \%)$ patients over a median follow up of 2.2 years (IQR 1.1-3.2). Pre-implant LVEF, paced QRS duration and RV pacing burden were independent predictors of PICM. Using 90\% specificity cut-off values for LVEF and paced QRS, and the value separating lowest tertile of RV pacing from the higher tertiles, three risk factors were identified: (i) baseline LVEF < 55\%, (ii) paced QRS duration $>160 \mathrm{msec}$, and (iii) RV pacing burden $>33 \%$. Patients with two or more risk factors were at the highest risk (OR 11.62, 95\% CI 4.62 - 29.21, p-value < 0.001) for developing PICM while those with one risk factor had an intermediate risk (OR 3.89, 95\% CI 1.62 - 9.34, p-value 0.002) when compared to those without any risk factors. Conclusion: Low-normal baseline LVEF, wider paced QRS and higher RV pacing burden independently predicted the development of PICM. The presence of [?]2 factors increased the odds of PICM, twelve-fold. Striving to get a narrower paced QRS, the only modifiable of the three risk factors, will help mitigate the development of PICM.
\end{abstract}

\section{Introduction:}

Conventional pacing from the right ventricle (RV) remains the most common site for cardiac pacing. But, RV pacing results in altered electromechanical activation of the ventricles which can have detrimental effects on myocardial perfusion, metabolism, and energy efficiency (1). This, in turn, can lead to progressive adverse remodelling at cellular and chamber levels with resultant deterioration in ventricular function (2). The term pacing-induced cardiomyopathy (PICM) was coined to identify the deleterious effects of RV pacing on left ventricular (LV) function and the resultant clinical syndrome of heart failure. Physiological pacing, with its advantage of minimizing or eliminating electromechanical dyssynchrony, is emerging as an attractive alternative (3). However, because of the steeper learning curve and paucity of long-term safety data, it is too early for it to completely replace conventional pacing (4). So, for the foreseeable future, RV pacing will remain the most common therapy for pacing indications.

Only a subset of patients with RV pacing develop PICM. Depending on the definition used, the incidence of PICM varies from 9 to $19.5 \%(5-8)$. The list of risk factors that predispose to PICM is not exhaustive and continues to evolve. One common factor across most studies is the burden of RV pacing $(7,9)$. South Asians have a high cardiovascular risk and younger age of hospitalization for heart failure (10). It is possible that ethnicity and demographic factors may play a role. The aim of this study was to determine the incidence and 
identify predictors of PICM in the South Asian population. We also sought to evaluate if these risk factors added up.

\section{Methods}

\subsection{Study Population}

Consecutive adult patients who underwent pacemaker (PM) in our institution from 2012 to 2018 were identified for analysis after institutional review board approval. Exclusion criteria were: (i) unavailability of echocardiogram done within 6 months before implantation, (ii) Left Ventricular ejection fraction (LVEF) $<50.0 \%$, (iii) significant valvular abnormalities (more than mild stenosis or regurgitation), (iv) complex congenital heart disease, (v) previous myocardial infarction or significant coronary artery disease, and (vi) implantable cardioverter-defibrillator, cardiac resynchronization therapy device, or single chamber atrial pacemaker implantation.

\subsection{Clinical Data and Measurements}

Data collection was done by reviewing the electronic medical records. Patient characteristics, indications, pre-implantation medical details, electrocardiographic (ECG), echocardiographic (ECHO) findings, and procedural details were reviewed. Intrinsic QRS duration was defined as the duration of conducted QRS (or escape QRS in case of no conducted beats) in the pre-implantation ECG. QRS complexes were classified according to duration and morphology using the standard criteria established by American Heart Association and Heart Rhythm Society expert consensus document (11). Ejection fraction(EF) was calculated using Simpson's method and other echocardiographic parameters were acquired according to American Society of Echocardiography and European Society of Echocardiography expert consensus document (12). The site of ventricular lead placement was ascertained from the procedural notes which was further confirmed from chest radiograph and classified as apical or non-apical based on the location. PM parameters were noted at discharge and at end of follow up period. RV pacing \% burden was recorded at end of follow up period or earlier if the primary outcome of PICM was reached. Paced QRS duration was measured from the immediate post pacemaker insertion electrocardiogram. PICM was defined as a fall in post implant LVEF by 10 percentage points to a LVEF of less than $50.0 \%$ from baseline due to RV pacing in the absence of other known causes of cardiomyopathy.

\subsection{Statistical Analysis}

Continuous variable distributions are expressed as means \pm standard deviation and median with interquartile range (IQR) and compared with Student's t-test or Mann-Whitney U test. Categorical variables are summarized as frequency and percentage and compared with the Chi-square test or Fisher's exact test. Patients lost to follow-up and missing values were excluded from the analysis. Pacing burden showed bimodal distribution and was categorized into three tertiles and analysed with the lowest tertile as the reference group. Multivariable analysis was done using binary logistic regression by selecting variables with p-value $<0.05$ on univariate analysis. Potential confounders previously found to have been associated with heart failure i.e. age, sex, presence of diabetes, presence of hypertension, beta-blocker use, and angiotensin-converting enzyme inhibitor use were also added to the model. The point estimates are reported as odds ratio (OR) and $95 \%$ confidence interval (CI). Receiver operator characteristics (ROC) analysis was performed on continuous variables found to be statistically significant on multivariable analysis. Categories were created based on the $90 \%$ specificity cut-off values of these variables. In the case of pacing burden, the 33rd percentile value separating the lowest tertile from the upper two tertiles was used as a cut-off. After these factors were identified, patients were categorized into those with no risk factors, one risk factor, and two or more risk factors. Binary logistic regression was performed with patients having none of the risk factors as the reference group. Interactions between these factors were not tested. A two-sided p-value of less than 0.05 was considered statistically significant. Statistical analysis was done with SPSS @ software (Ver. 16.0, IBM, USA).

\section{Results}

\subsection{Patient and pacing characteristics}


A total of 1132 underwent PM implantation during the study period. Of these, 134 patients with preimplantation LVEF < 50.0\% were excluded. Another 249 (24.9\%) patients were lost to follow-up and were excluded. Hence, a total of 749 patients were included in the final analysis.

The mean age of the cohort was $59.2 \pm 14.1$ years and $31.6 \%$ were female. The most common indication for pacing was degenerative high-grade AV block seen in $74.37 \%$ of the study population. The other causes included sick sinus syndrome, carotid sinus hypersensitivity and syncope of unknown origin. About half of the patients had baseline bundle branch block or intraventricular conduction defect (IVCD). Mean preimplantation LVEF was $57.3 \pm 3.3 \%$. The majority of the patients $(71.2 \%)$ received a dual-chamber pacemaker and most $(69.6 \%)$ had an RV lead placed in a non-apical location. The median RV pacing burden was $81 \%$ (IQR 15-99\%). Other patient, procedural, and pacing characteristics are summarized in Table 1.

\subsection{Outcome}

Over a median follow-up of 2.2 (IQR: 1.1-3.2) years, 74/749 (9.9\%) patients developed PICM. In patients who developed PICM, LVEF decreased from a mean $56.6+/-3.35 \%$ to $41.5+/-6.87 \%$. The earliest incidence of PICM was at 6 months and the longest was at 6 years following implantation. Patients who developed PICM had a lower pre-implantation EF compared to those who did not develop PICM $(56.6 \pm 3.0 \% \mathrm{Vs}$ $57.5 \pm 2.6$; p-value 0.005) and higher RV pacing burden (median 98.2\% [IQR $80.5-99.0 \%$ ] Vs $75.0 \%$ [IQR 13.0 - $99.0 \%$ ]; p-value $<0.001)$.

\subsection{Right ventricular pacing burden and outcome}

The median RV pacing for the whole cohort was 81.0\% (IQR 15.0 - $99.0 \%$ ). Most patients were in the extreme categories of pacing burden - $21.4 \%$ having [?] $10.0 \%$ RV pacing and $43.5 \%$ were paced $>90.0 \%$ (Figure 1.a). So, the cohort was divided into three tertiles based on RV pacing percentage of 34.0\% (33rd percentile) and $98.0 \%$ (67th percentile). The incidence of PICM in the first, second and third tertiles was $3.8 \%, 12.7 \%$, and $14.6 \%$, respectively (Chi-square p-value for trend $<0.001$; figure $1 . b$ ).

\subsection{Predictors of pacing-induced cardiomyopathy}

On univariate analysis, significant predictors of PICM were lower baseline LVEF (OR 0.85 per 1\% decrease, 95\% CI 0.76 - 0.95, p-value 0.004), paced QRS duration (OR 1.34 per 10 msec increase, $95 \%$ CI $1.22-1.48$, p-value $<0.001)$, and RV pacing burden (Table. 2). Patients in the highest tertile of RV pacing (>98.0\% RV pacing) were at highest risk (OR 4.31, 95\% CI $2.02-9.18$, p-value < 0.001) compared to those in the lowest tertile (reference group; $<34.0 \%$ RV pacing). Patients in the middle tertile $(34.0-98.0 \%$ pacing) had intermediate risk (OR 3.67, 95\% CI 1.70 - 7.92, p-value < 0.001).

Multivariable analysis was performed by adding the variables that reached statistical significance on univariate analysis and those which were considered potential confounders i.e. age, sex, presence of diabetes, hypertension, beta-blocker, and angiotensin converting enzyme (ACE) inhibitor use, to the model (table.2). After adjustment for other variables, baseline LVEF (adjusted OR 0.87 per 1\% increase, 95\% CI 0.77 - 0.97 , p-value 0.016), paced QRS duration (adjusted OR 1.22 per 10msec increase, 95\% CI 1.10 - 1.48, p-value < 0.001 ), and RV pacing burden remained significant (Table 2).

\subsection{Number of risk factors and the risk of PICM}

Receiver operator characteristics (ROC) analysis was done for baseline LVEF and paced QRS duration to determine the cut-offs to predict PICM. Area under curve (AUC) for LVEF was 0.61 (95\% CI 0.53 - 0.68, p-value 0.003) and paced QRS duration was 0.71 (95\% CI $0.65-0.77$, p-value $<0.001)$. The cut-off value with $90.0 \%$ specificity to predict PICM was $55.0 \%$ for LVEF and $160 \mathrm{msec}$ for QRS duration. For RV pacing burden, a value of $34.0 \%$ (cut-off between the lowest tertile and the higher tertiles) was taken. Based on these cut-off values, the risk factors were: (i) baseline LVEF $<55.0 \%$, (ii) paced QRS duration $>160 \mathrm{msec}$, and (iii) RV pacing burden $>34.0 \%$. Patients with two or more of the above risk factors were at highest risk (OR 11.62, 95\% CI $4.62-29.21$, p-value < 0.001) of developing PICM compared to those without any 
of the risk factors (reference group; figure.2). Those with one of the risk factors were at intermediate risk (OR 3.89, 95\% CI 1.62 - 9.34, p-value 0.002).

\section{Discussion}

The main findings of the current study are : (i) PICM occurred in about $10 \%$ of this cohort of south Asian population, (ii) lower pre-implant LVEF, wider paced QRS duration, and higher RV pacing burden were independent predictors of PICM, and (iii) a combination of [?] 2 of these risk factors increases the odds of developing PICM by 12 times compared to those who do not have any of the factors.

The observed incidence of PICM over long term follow up in our study was slightly lower compared to that from previous studies which ranged from $9 \%$ to $19.5 \%$ (5-8). This is probably due to heterogeneous definitions and methodological issues. Zhang et al reported a higher incidence of PICM of 26\%, but they did not identify and exclude patients with alternative aetiologies of heart failure (13). Possible explanations for the lower incidence of PICM in our study include lesser overall RV pacing (>40\% RV pacing was noted only in $65 \%$ of the cohort) and relatively short follow up (earlier reports show that risk of incident PICM persists years after exposure, sometimes as late as 15 years (6)). Other contributing factors include exclusion of patients having less than 6 months follow up (PICM can develop within 1 month of implantation (5)) and lesser use of apical pacing.

Kiehl et al's multivariate analysis showed lower pre-PM LVEF ( HR: 1.047 per 1\% LVEF decrease, p=0.42) as a statistically significant factor for the development of PICM (7). In our study too the results were similar $(\mathrm{p}=0.016)$. It is however still unclear as to whether each incremental percentage increase in LVEF above $50 \%$ is protective until it reaches a particular threshold.

The benefit of biventricular pacing resulting in a narrow QRSd and restoring cardiac synchrony in patients with LBBB and QRSd $>150 \mathrm{msec}$ has been well established (14). Miyoshi et al in a prospective analysis of 92 patients with permanent RV apical pacing predicted the development of symptomatic heart failure (46.6\% vs $11.6 \%, \mathrm{p}<0.05)$ and worsening LV parameters using a pQRSd cut-off value of $>190 \mathrm{msec}(15)$. PREDICT-HF was an observational cohort study which showed an incidence of heart failure of $56.8 \%$ with pQRSd $>190 \mathrm{msec}$ compared to $9.4 \%$ in patients with pQRSd $<160 \mathrm{msec}(\mathrm{p}<0.001)(16)$. Our study too showed pQRSd to be an independent predictor and cut off values of $>125 \mathrm{msec}$ had best sensitivity $(90 \%)$ and $>160 \mathrm{msec}$ had best specificity $(90 \%)$ for development of PICM. These findings demonstrate that patients with a higher pQRSd need to undergo active and close follow up with more periodic echocardiography. As a corollary, patients with a low pQRSd can be followed up less rigorously which would be beneficial in a resource poor setting. Sharma et al demonstrated this in a prospective study where those with a narrow pQRSd had preserved LVEF at one year follow up using equilibrium radionucleotide angiography (17). This data further supports the concept that wide pQRSd is a marker of electrical dyssynchrony which leads to adverse remodelling causing PICM in patients with RV pacing. Hence striving to achieve a narrow QRS during the time of implantation could be the only modifiable risk factor that leads to better outcomes with respect to PICM. The advent of His and Left bundle pacing, result in less electromechanical dyssynchrony and are likely to replace the traditional pacing modalities in future $(3,4)$.

RV pacing of more than $40 \%$ is the suggested threshold for the development of PICM by the MOST and DAVID trials (9). Though more than $65 \%$ of our study cohort had a RV pacing percentage of more than $40 \%$, PICM occurred in some patients who were paced less than this threshold. Kiehl et al showed that RV pacing percentage independently predicted PICM both as a continuous variable (HR: 1.01 per 1\% RV pacing, 95\% CI: $1.002-1.02, \mathrm{p}=0.02)$ and even more significantly as a categorical variable $(<20 \%$ or $[?] 20 \%$ RV pacing) (HR: 6.76, 95\% CI: 2.08-22.0, $\mathrm{p}=0.002$ ) (7). Hence it should be noted that even in patients with low RV pacing percentages there is a need to consider the possibility of future occurrence of PICM as noted in our study. The odds of them developing PICM though low will still warrant periodic monitoring. This has significant cost implications for developing countries where the patients have to bear their own medical expenses.

A meta-analysis published in 2012 suggested that patients with RV non apical pacing may have higher LVEF 
on follow up compared to those with RV apical pacing (18). Randomised controlled trials comparing the two modes of RV pacing showed no difference in levels of brain natriuretic peptide levels, ejection fraction or exercise capacity over a follow up period of 18 months(19,20). The PROTECT-PACE trial which was planned towards identifying the optimal pacing site to reduce PICM showed no benefit of non-apical pacing on LV function over a 2 year follow up period (21). In our study too there was no significant difference between apical and non-apical positions for RV pacing towards the occurrence of PICM $(p=0.9)$. Similar to our findings Chan et al reported that LV volume and systolic function are better predicted by pQRSd and not the pacing site, with pQRSd being a target for pacing site optimisation (22).

The native QRS duration (nQRSd) is predominantly influenced by the level of antegrade delay in the His Purkinje system during atrioventricular block. Khurshid et al in a single centre study in 2016 showed that nQRSd (HR:1.03 per msec: $\mathrm{P}<0.001$ ) is an independent predictor for PICM (23). Pap et al showed that $\mathrm{nQRSd}$ is a good predictor of pQRSd in patients with AV block and normal LVEF undergoing PM implantation (24). Though our study did not find nQRSd as a significant risk factor for PICM; the p value suggests that with larger numbers this may not be true. A wider nQRSd prior to implantation of PM in patients with normal LVEF implies greater electrical dyssynchrony which over time translates to mechanical dyssynchrony. The latter perhaps predisposes them to PICM with the added insult of RV pacing.

Our study for the first time looked at whether the risk factors that predicted PICM added up and found that they did. Prescence of more than one risk factor was incrementally additive. Of the 3 risk factors: pre implant LVEF, RV pacing burden and pQRSd; only the latter was modifiable. Hence at implantation it would be worthwhile investing time in choosing a location where the pQRSd is the lowest possible.

\section{Strengths and Weaknesses:}

One of the main strengths of our study is a large sized contemporary cohort of patients from South Asia, who went through a thorough process of recruitment and have comprehensive data for analysis. This is the first study to not only identify the risk factors for PICM but also give an additive risk assessment. This would significantly help the identification of patients prone to developing this condition.

There were a few limitations noted in this study. First, though extensive data search was done to identify and exclude all other potential sources of cardiomyopathy, it is possible that some patients diagnosed with PICM may have had an alternate cause for decrease in LV function. However, this is what is commonly observed in clinical practice as PICM is a diagnosis of exclusion. The other limitation was significant longterm loss of follow up. This may have led to an inclusion bias with symptomatic patients in heart failure being more likely to follow up and hence being included in the study. This, however, is unlikely considering the incidence of PICM in our study is comparable if not lower than that in studies done elsewhere. We evaluated if the risk factors predisposing to PICM added up, but our numbers were not large enough to rule out interactions between them. Some individuals develop PICM despite lacking these suggesting that there are other predisposing factors that are hitherto unknown.

\section{Conclusion:}

In patients undergoing permanent PM, low-normal baseline LVEF, wider paced QRS duration and a higher RV pacing burden independently predicted the development of PICM in this cohort from South Asia. Presence of these risk factors was incrementally additive with a patient having 2 or more of these risk factors

having twelve-fold increased odds of developing PICM. Striving to reduce the only modifiable risk factor the pQRSd at the time of implant, could help reduce the incidence of PICM.

\section{Abbreviations:}

RV: right ventricular, LVEF: left ventricular ejection fraction, PICM: pacing induced cardiomyopathy, PM: permanent pacemaker implantation, LV: left ventricular

\section{Bibliography}


1. Tse H-F, Lau C-P. Long-Term Effect of Right Ventricular Pacing on Myocardial Perfusion and Function. J Am Coll Cardiol. 1997 Mar 15;29(4):744-9.

2. Karpawich PP, Justice CD, Cavitt DL, Chang CH. Developmental sequelae of fixed-rate ventricular pacing in the immature canine heart: an electrophysiologic, hemodynamic, and histopathologic evaluation. Am Heart J. 1990 May;119(5):1077-83.

3. Das A, Kahali D. Physiological cardiac pacing: Current status. Indian Heart J. 2016;68(4):552-8.

4. Vijayaraman P, Bordachar P, Ellenbogen KA. The Continued Search for Physiological Pacing: Where Are We Now? J Am Coll Cardiol. 2017 Jun 27;69(25):3099-114.

5. Khurshid S, Epstein AE, Verdino RJ, Lin D, Goldberg LR, Marchlinski FE, et al. Incidence and predictors of right ventricular pacing-induced cardiomyopathy. Heart Rhythm Off J Heart Rhythm Soc. 2014 Sep;11(9):1619-25.

6. Dreger H, Maethner K, Bondke H, Baumann G, Melzer C. Pacing-induced cardiomyopathy in patients with right ventricular stimulation for \&gt;15 years. Europace. 2012 Feb 1;14(2):238-42.

7. Kiehl EL, Makki T, Kumar R, Gumber D, Kwon DH, Rickard JW, et al. Incidence and predictors of right ventricular pacing-induced cardiomyopathy in patients with complete atrioventricular block and preserved left ventricular systolic function. Heart Rhythm. 2016;13(12):2272-8.

8. Kim JH, Kang K-W, Chin JY, Kim T-S, Park J-H, Choi YJ. Major determinant of the occurrence of pacing-induced cardiomyopathy in complete atrioventricular block: a multicentre, retrospective analysis over a 15-year period in South Korea. BMJ Open. 2018 Feb 1;8(2):e019048.

9. Sharma AD, Rizo-Patron C, Hallstrom AP, O'Neill GP, Rothbart S, Martins JB, et al. Percent right ventricular pacing predicts outcomes in the DAVID trial. Heart Rhythm. 2005 Aug;2(8):830-4.

10. Singh N, Gupta M. Clinical characteristics of South Asian patients hospitalized with heart failure. Ethn Dis. 2005;15(4):615-9.

11. Surawicz B, Childers R, Deal BJ, Gettes LS. AHA/ACCF/HRS Recommendations for the Standardization and Interpretation of the Electrocardiogram: Part III: Intraventricular Conduction Disturbances: A Scientific Statement From the American Heart Association Electrocardiography and Arrhythmias Committee, Council on Clinical Cardiology; the American College of Cardiology Foundation; and the Heart Rhythm Society: Endorsed by the International Society for Computerized Electrocardiology. Circulation. 2009 Mar 17;119(10):e235-40.

12. Lang RM, Badano LP, Mor-Avi V, Afilalo J, Armstrong A, Ernande L, et al. Recommendations for cardiac chamber quantification by echocardiography in adults: an update from the American Society of Echocardiography and the European Association of Cardiovascular Imaging. J Am Soc Echocardiogr Off Publ Am Soc Echocardiogr. 2015 Jan;28(1):1-39.e14.

13. Zhang X-H, Chen H, Siu C-W, Yiu K-H, Chan W-S, Lee KL, et al. New-onset heart failure after permanent right ventricular apical pacing in patients with acquired high-grade atrioventricular block and normal left ventricular function. J Cardiovasc Electrophysiol. 2008 Feb;19(2):136-41.

14. Moss AJ, Hall WJ, Cannom DS, Klein H, Brown MW, Daubert JP, et al. Cardiac-Resynchronization Therapy for the Prevention of Heart-Failure Events. N Engl J Med. 2009 Oct 1;361(14):1329-38.

15. Prolonged Paced QRS Duration as a Predictor for Congestive Heart Failure in Patients with Right Ventricular Apical Pacing - MIYOSHI - 2005 - Pacing and Clinical Electrophysiology - Wiley Online Library [Internet]. [cited 2020 Apr 27]. Available from: https://onlinelibrary.wiley.com/doi/abs/10.1111/j.15408159.2005.50181.x

16. Chen S, Yin Y, Lan X, Liu Z, Ling Z, Su L, et al. Paced QRS duration as a predictor for clinical heart failure events during right ventricular apical pacing in patients with idiopathic complete atrioventricular 
block: results from an observational cohort study (PREDICT-HF). Eur J Heart Fail. 2013 Mar;15(3):352-9.

17. Sharma G, Shetkar SS, Patel CD, Singh H, Naik N, Roy A, et al. Paced QRS duration predicts left ventricular function in patients with permanent pacemakers - One-year follow-up study using equilibrium radionuclide angiography (ERNA). Indian Pacing Electrophysiol J. 2015 Jul 13;15(2):90-5.

18. Shimony A, Eisenberg MJ, Filion KB, Amit G. Beneficial effects of right ventricular non-apical vs. apical pacing: a systematic review and meta-analysis of randomized-controlled trials. EP Eur. 2012 Jan $1 ; 14(1): 81-91$.

19. Kypta A, Steinwender C, Kammler J, Leisch F, Hofmann R. Long-term outcomes in patients with atrioventricular block undergoing septal ventricular lead implantation compared with standard apical pacing. Eur Eur Pacing Arrhythm Card Electrophysiol J Work Groups Card Pacing Arrhythm Card Cell Electrophysiol Eur Soc Cardiol. 2008 May;10(5):574-9.

20. Nikoo MH, Ghaedian MM, Kafi M, Fakhrpour A, Jorat MV, Pakfetrat M, et al. Effects of right ventricular septal versus apical pacing on plasma natriuretic peptide levels. J Cardiovasc Dis Res. 2011;2(2):104-9.

21. Kaye GC, Linker NJ, Marwick TH, Pollock L, Graham L, Pouliot E, et al. Effect of right ventricular pacing lead site on left ventricular function in patients with high-grade atrioventricular block: results of the Protect-Pace study. Eur Heart J. 2015 Apr 7;36(14):856-62.

22. Chan N-Y, Yuen H-C, Choy C-C, Mok N-S, Tsui P-T, Lau C-L, et al. Left ventricular volumes and systolic function after long-term right ventricular pacing may be predicted by paced QRS duration, but not pacing site. Heart Lung Circ. 2014 Jan;23(1):43-8.

23. Khurshid S, Liang JJ, Owens A, Lin D, Schaller R, Epstein AE, et al. Longer Paced QRS Duration is Associated With Increased Prevalence of Right Ventricular Pacing-Induced Cardiomyopathy. J Cardiovasc Electrophysiol. 2016 Oct;27(10):1174-9.

24. Pap R, Furge P, Bencsik G, Makai A, Saghy L, Forster T. Native QRS complex duration predicts paced QRS width in patients with normal left ventricular function and right ventricular pacing for atrioventricular block. J Electrocardiol. 2007 Jul 1;40(4):360-4.

Table 1. Baseline patient and pacemaker characteristics.

\begin{tabular}{|c|c|c|c|c|}
\hline & Total $(n=749)$ & $\operatorname{PICM}(n=74)$ & $\begin{array}{l}\text { No PICM } \\
(\mathrm{n}=675)\end{array}$ & P Value ${ }^{*}$ \\
\hline Age, years & $59.19 \pm 14.08$ & $57.7 \pm 15.67$ & $59.36 \pm 13.90$ & 0.338 \\
\hline Female (\%) & $237(31.6)$ & $25(33.8)$ & $212(31.4)$ & 0.676 \\
\hline Diabetes (\%) & $257(34.36)$ & $24(32.4)$ & $233(34.6)$ & 0.713 \\
\hline $\begin{array}{l}\text { Hypertension } \\
(\%)\end{array}$ & $406(54.28)$ & $41(55.4)$ & $365(54.1)$ & 0.838 \\
\hline $\begin{array}{l}\text { Hypothyroidism } \\
\text { (\%) }\end{array}$ & $84(11.23)$ & $10(13.5)$ & $74(10.9)$ & 0.512 \\
\hline $\begin{array}{l}\text { Drugs }(\%) \\
\text { Beta-blocker } \\
\text { ACE inhibitors }\end{array}$ & $\begin{array}{l}229(30.57) 292 \\
(38.99)\end{array}$ & $\begin{array}{l}28(37.8) 34 \\
(45.9)\end{array}$ & $\begin{array}{l}201(29.8) 258 \\
(38.2)\end{array}$ & 0.1530 .196 \\
\hline $\begin{array}{l}\text { Indication (\%) } \\
\text { High Grade AV } \\
\text { Block Sinus Node } \\
\text { Dysfunction } \\
\text { Others }\end{array}$ & $\begin{array}{l}557(74.37) 180 \\
(24.03) 12(1.60)\end{array}$ & $\begin{array}{l}65(87.84) 8(10.81) \\
1(1.35)\end{array}$ & $\begin{array}{l}492(72.89) 172 \\
(25.48) 11(1.63)\end{array}$ & 0.013 \\
\hline
\end{tabular}




\begin{tabular}{|c|c|c|c|c|}
\hline $\begin{array}{l}\text { Implanting } \\
\text { physician }(\%) \\
\text { More than } 3 \text { yr } \\
\text { experience Less } \\
\text { than } 3 \text { year } \\
\text { experience }\end{array}$ & $\begin{array}{l}451(60.21) 298 \\
(39.79)\end{array}$ & $45(60.8) 29(39.2)$ & $\begin{array}{l}406(60.15) 269 \\
(39.85)\end{array}$ & 0.912 \\
\hline Pacing mode (\%) & $65(8.68) 18(2.40)$ & $4(5.4) 2(2.7) 8(10.8)$ & $61(9) 16(2.37)$ & 0.855 \\
\hline $\begin{array}{l}\text { VVI VVIR VDD } \\
\text { DDD DDDR }\end{array}$ & $\begin{array}{l}69(9.21) 133 \\
(17.76) 464(61.95)\end{array}$ & $14(18.9) 46(62.16)$ & $\begin{array}{l}61(9.04) 119(17.6) \\
418(61.9)\end{array}$ & \\
\hline $\begin{array}{l}\text { Rate adaptive } \\
\text { pacing }(\%)\end{array}$ & $587(78.37)$ & $59(79.7)$ & $528(78.2)$ & 0.765 \\
\hline $\begin{array}{l}\text { Apical location } \\
(\%)\end{array}$ & $228(30.44)$ & $23(31.1)$ & $205(30.8)$ & 0.900 \\
\hline $\begin{array}{l}\text { Baseline LV EF } \\
\%\end{array}$ & $57.3 \pm 3.3$ & $56.1 \pm 1.4$ & $57.1 \pm 2.1$ & 0.047 \\
\hline $\begin{array}{l}\text { Intrinsic QRS } \\
\text { duration, msec }\end{array}$ & $122.9 \pm 26.2$ & $127.4 \pm 25.9$ & $121.7 \pm 26.2$ & 0.088 \\
\hline $\begin{array}{l}\text { Intrinsic QRS } \\
\text { Morphology (\%) } \\
\text { Narrow QRS } \\
\text { RBBB/ IVCD } \\
\text { LBBB }\end{array}$ & 50.4324 .7924 .79 & 46.418 .834 .8 & 50.8725 .4423 .69 & 0.109 \\
\hline $\begin{array}{l}\text { Paced QRS } \\
\text { duration, msec }\end{array}$ & $134.9 \pm 25.4$ & $149.8 \pm 20.4$ & $133.25 \pm 25.3$ & 0.023 \\
\hline $\begin{array}{l}\text { Median RV } \\
\text { pacing burden } \\
\%,(\text { IQR })\end{array}$ & $81(15-99)$ & $98.15(81-99)$ & $75(13-99)$ & 0.0006 \\
\hline
\end{tabular}

Note: Values are mean \pm standard deviation, frequency $(\%)$ or median (25th percentile -75 th percentile)

* P Value for comparison between PICM and no PICM groups

Table.2. Predictors of pacing induced cardiomyopathy

\begin{tabular}{|c|c|c|c|c|c|c|}
\hline & $\begin{array}{l}\text { PICM }(\mathrm{n}= \\
74)\end{array}$ & $\begin{array}{l}\text { No PICM } \\
\mathbf{n}=675)\end{array}$ & $\begin{array}{l}\text { Univariate } \\
\text { OR }(95 \% \\
\text { CI) }\end{array}$ & $\mathbf{P}$ value & $\begin{array}{l}\text { Adjusted } \\
\text { OR }(95 \% \\
\text { CI })\end{array}$ & $\mathbf{P}$ value \\
\hline Age, years & $57.7 \pm 15.7$ & $59.4 \pm 13.9$ & $\begin{array}{l}0.99(0.98- \\
1.01)\end{array}$ & 0.338 & $\begin{array}{l}0.99(0.97- \\
1.01)\end{array}$ & 0.422 \\
\hline $\begin{array}{l}\text { Female sex } \\
(\%)\end{array}$ & $25(33.8)$ & $212(31.4)$ & $\begin{array}{l}1.11(0.67- \\
1.85)\end{array}$ & 0.677 & $\begin{array}{l}1.34(0.77- \\
2.34)\end{array}$ & 0.297 \\
\hline $\begin{array}{l}\text { Diabetes } \\
\text { mellitus } \\
(\%)\end{array}$ & $24(32.4)$ & $233(34.6)$ & $\begin{array}{l}1.10(0.66- \\
1.84)\end{array}$ & 0.713 & $\begin{array}{l}0.82(0.46- \\
1.45)\end{array}$ & 0.494 \\
\hline $\begin{array}{l}\text { Hypertension } \\
\text { (\%) }\end{array}$ & $41(55.4)$ & $365(54.2)$ & $\begin{array}{l}0.95(0.59- \\
1.54)\end{array}$ & 0.838 & $\begin{array}{l}1.01(0.56 \\
-1.85)\end{array}$ & 0.97 \\
\hline $\begin{array}{l}\text { Beta- } \\
\text { blocker use } \\
(\%)\end{array}$ & $28(37.8)$ & $201(29.8)$ & $\begin{array}{l}1.29(0.71- \\
2.34)\end{array}$ & 0.410 & $\begin{array}{l}1.31(0.76- \\
2.26)\end{array}$ & 0.335 \\
\hline $\begin{array}{l}\text { ACE } \\
\text { inhibitor } \\
\text { use (\%) }\end{array}$ & $34(45.9)$ & $258(38.2)$ & $\begin{array}{l}1.20(0.68- \\
2.12)\end{array}$ & 0.535 & $\begin{array}{l}1.15(0.67- \\
1.97)\end{array}$ & 0.608 \\
\hline
\end{tabular}




\begin{tabular}{|c|c|c|c|c|c|c|}
\hline $\begin{array}{l}\text { Baseline } \\
\text { LVEF }^{*}, \%\end{array}$ & $56.6 \pm 3.0$ & $57.5 \pm 2.6$ & $\begin{array}{l}0.85(0.76- \\
0.95)\end{array}$ & 0.004 & $\begin{array}{l}0.87(0.77- \\
0.97)\end{array}$ & 0.016 \\
\hline $\begin{array}{l}\text { Paced } \\
\text { QRS } \\
\text { duration, } \\
\text { msec }\end{array}$ & $149.8 \pm 20.4$ & $133.2 \pm 25.3$ & $\begin{array}{l}1.34(1.22- \\
1.48)\end{array}$ & $<0.001$ & $\begin{array}{l}1.22(1.10- \\
1.48)\end{array}$ & $<0.001$ \\
\hline $\begin{array}{l}\text { RV pacing } \\
\text { burden } \\
\text { Tertile } 1 \text { ( } \\
<34 \%) \\
\text { Tertile 2 } \\
(34-98 \%) \\
\text { Tertile } 3 \\
(>98 \%)\end{array}$ & $\begin{array}{l}9(12.2) 30 \\
(40.5) 35 \\
(47.3)\end{array}$ & $\begin{array}{l}227(35.6) \\
206(32.2) \\
205(32.1)\end{array}$ & $\begin{array}{l}\text { Reference } \\
3.67(1.70- \\
7.92) 4.31 \\
(2.02-9.18)\end{array}$ & $\begin{array}{l}-0.001< \\
0.001\end{array}$ & $\begin{array}{l}\text { Reference } \\
2.48(1.11- \\
5.54) 2.46 \\
(1.11-5.46)\end{array}$ & - 0.0270 .027 \\
\hline
\end{tabular}

Note: Values are mean \pm standard deviation, frequency $(\%)$ or median (25th percentile -75 th percentile)

* OR for every $1.0 \%$ increase in EF

OR for every $10 \mathrm{msec}$ increase in paced QRS
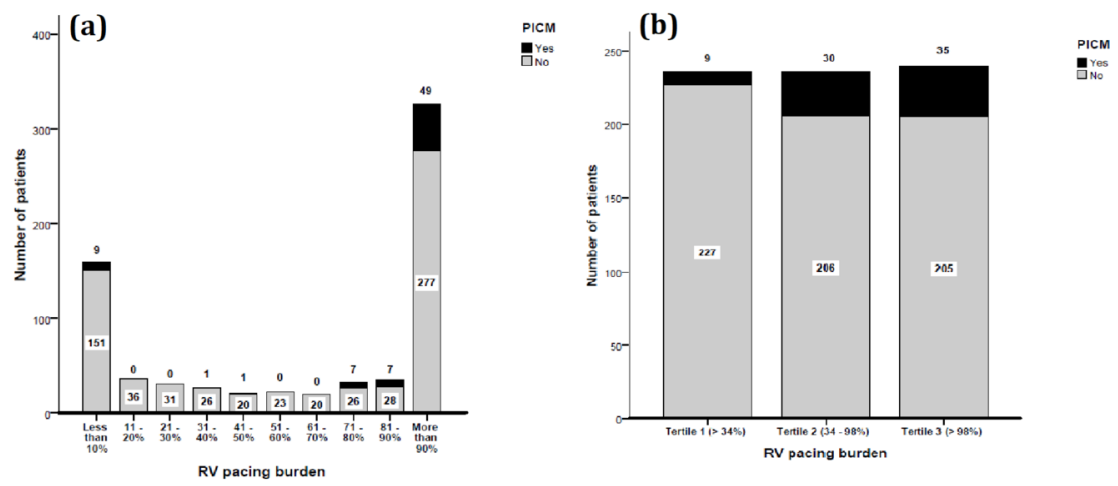

Figure. 1.Right Ventricular pacing burden and Outcome. 


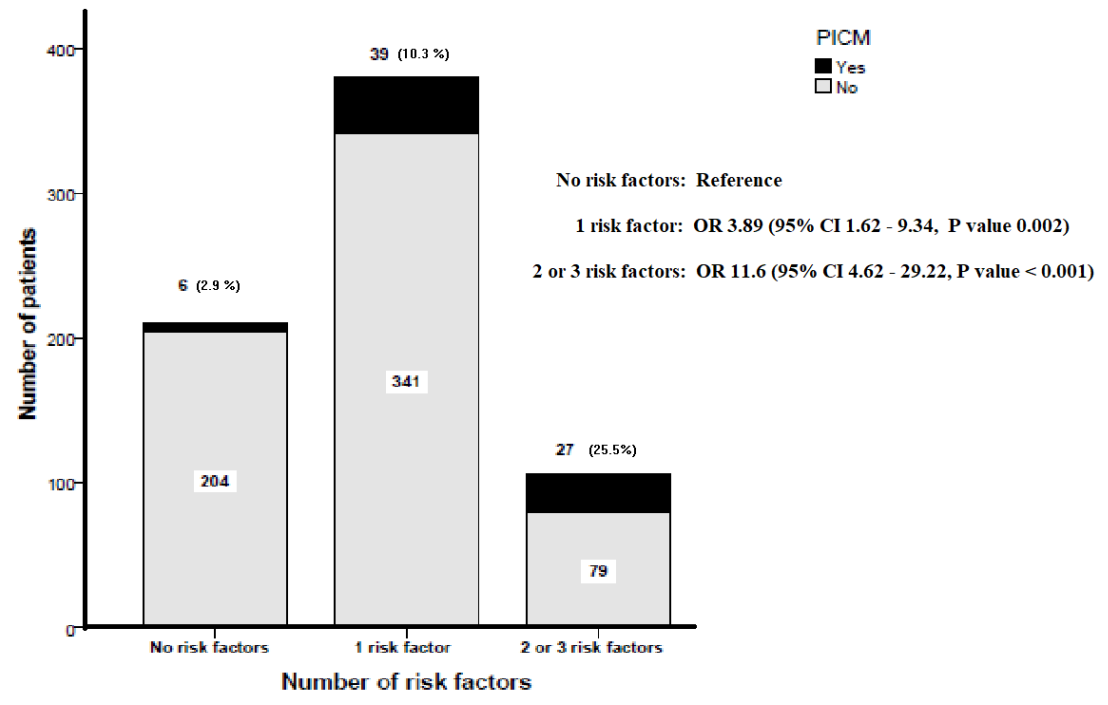

Figure.2.

Number of risk factors and risk of PICM.

\section{Hosted file}

Tables JCE submission.docx available at https://authorea.com/users/355471/articles/478682-dothe-predictors-of-right-ventricular-pacing-induced-cardiomyopathy-add-up
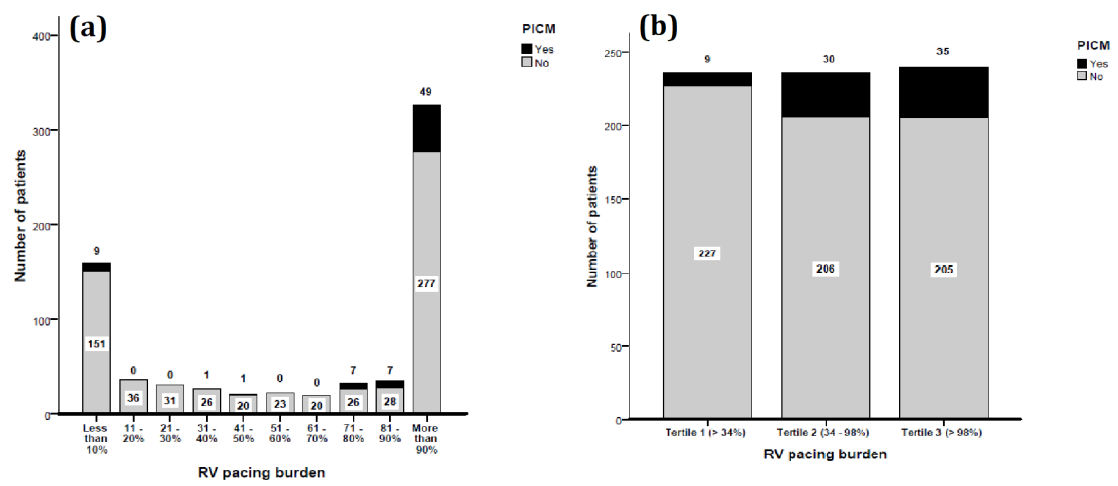


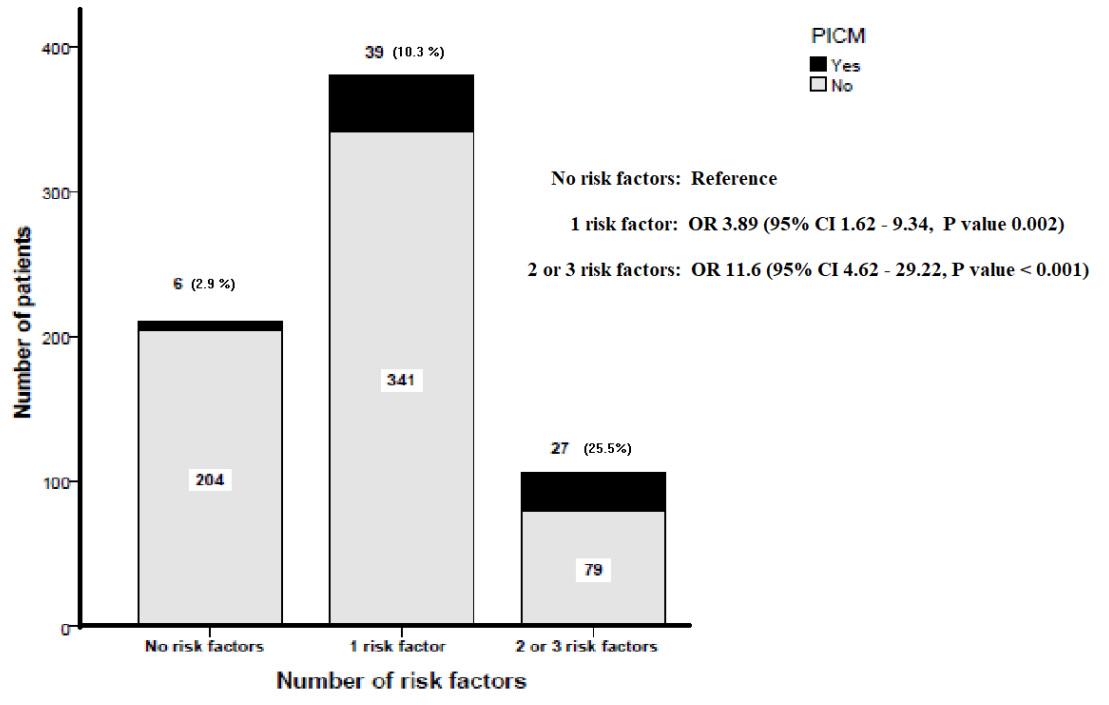

